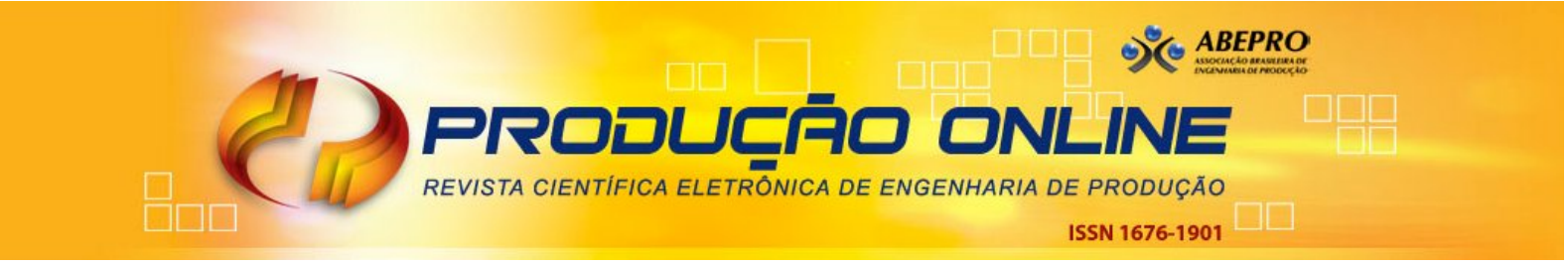

\title{
MAPEAMENTO DO FLUXO DE VALOR E USO DA SIMULAÇÃO INTEGRADA LEAN COM SISTEMAS CIBER-FÍSICOS EM UMA INDÚSTRIA DE EMBALAGENS FLEXÍVEIS
}

\section{VALUE STREAM MAPPING AND THE USE OF INTREGRATED SIMULATION LEAN AND CYBER-PHYSICAL SYSTEMS IN A FLEXIBLE'S PACKAGING INDUSTRY}

\author{
Danilo Ribamar Sá Ribeiro* E-mail: danilo saribeiro@hotmail.com \\ Mariele Canal Bonfante* E-mail: marielebonfante@gmail.com \\ Enzo Morosini Frazzon* E-mail: enzo.frazzon@ufsc.br \\ Fernando Antônio Forcellini E-mail: fernando.forcellini@ufsc.br \\ * Universidade Federal de Santa Catarina (UFSC), Florianópolis, SC
}

Resumo: A utilização conjunta de MFV e Simulação tem ganhando cada vez mais espaço, apresentando-se como ferramentas essenciais ao contribuir com a proposição de novos cenários para facilitar a implementação de melhorias, apoiada pela difusão de tecnologias de informação e pela contínua adoção dos conceitos de Sistemas Ciber-Físicos (CPS). Nesse contexto, esta pesquisa teve como objetivo propor e analisar, por meio da aplicação da ferramenta de Mapeamento de Fluxo de Valor e simulação, a adoção de CPS no fluxo de uma linha de produção de bobina de filme laminado, considerada como produto mais representativo no volume de produção de uma empresa produtora de embalagens flexíveis. Ademais, compara-se o cenário do Estado Atual da linha e um cenário do Estado Futuro de integração Lean com CPS, que foram construídos com o auxílio do software SIMIO ${ }^{\circledR}$. Os resultados mostraram que a integração das ferramentas de simulação de produção e do MFV permitiram desenvolver um modelo virtual de produção, que além de representar o fluxo real da produção é capaz de analisar cenários de otimização das operações da manufatura, bem como apresenta as vantagens da adoção de sistemas inteligentes na linha produtiva.

Palavras-chave: Mapeamento do Fluxo de Valor. Análise baseada Simulação. Lean. CPS.

\begin{abstract}
The integrated use of VSM and Simulation is increasing as essential tools to contribute with the proposition of new scenarios to guide the implementation of improvements. It is supported by the diffusion of $\mathrm{TI}$ and by the continuous adoption of the concepts of CPS. In this context, this paper aims to propose and analyze the adoption of CPS in the flow of a laminated film coil's production line, through the application of the VSM and Simulation. This is the most representative product in the volume of production of a company producing flexible packaging. In addition, the Current and Future States (integration Lean with CPS) scenarios are compared, which were constructed using SIMIO ${ }$ software. The results showed that the integration of the production simulation and the VSM tool allowed to develop a virtual model of production, which besides representing the actual flow of the production is able to analyze scenarios of optimization of the manufacture's operations, as well as presents the advantages of the adoption of intelligent systems in the production line.
\end{abstract}

Keywords: Value Stream Mapping. Simulation based analysis. Lean. CPS. 


\section{INTRODUÇÃO}

Atualmente, observa-se que devido ao aumento da concorrência, as organizações necessitam apresentar estratégias competitivas diante do mercado. Para isso, além de possuir um bom produto que atenda às necessidades dos clientes, as empresas de manufatura precisam de processos que melhorem sua produtividade e diminuam seus custos operacionais, além da capacidade de atender a demanda de mercado (SILVA, 2013; MELLO e PAVAN, 2017).

A manufatura representa uma das mais importantes aplicações da simulação, pois esta técnica é uma ferramenta valiosa usada por engenheiros para avaliar os efeitos do capital investido em equipamentos e instalações físicas. A Análise baseada em simulação pode ser utilizada para prever o desempenho de um sistema planejado e comparar soluções alternativas para algum problema de projeto (BENEDETTINI e TJAHJONO, 2009).

Concomitante com a simulação, a ferramenta Mapeamento do Fluxo de Valor (MFV) oriunda da Abordagem Lean contribuem nesse processo de identificação e mitigação de desperdícios ao promover que a empresa se torne mais competitiva (SILVA, 2013). O MFV é uma ferramenta de aprimoramento empresarial que auxilia na visualização de todo o processo de produção, representando fluxo de material e informação (SINGH et al., 2013).

Desta forma, a simulação pode ser empregada em ambientes fabris para facilitar as tomadas de decisões sem que para isso haja mudanças no processo produtivo. Em vista disso, as melhorias propostas para atingir um processo enxuto seriam simuladas antes de qualquer mudança. Outra vantagem seria a simulação de diversos cenários, o que facilitaria aos gestores a implementação das melhores práticas (OLIVEIRA, 2008; COSTA, 2011; SILVA, 2013).

Silva (2013) afirma que ainda são poucos os trabalhos que utilizam a simulação nessa área com o objetivo de propor novos cenários para facilitar a implementação do mapa futuro.

Neste contexto, o uso de sistemas inteligentes integrando TI (Tecnologia de Informações) e CPS (Cyber-Physical Systems - Sistemas Ciber-Físicos) pode promover a eficiência na gestão de informações e controle para estes processos. No caso, o TI captura e gerencia os dados e, por sua vez, o CPS utiliza o poder de 
computação para monitorar e fazer alterações em processos físicos, e estes por sua vez realimentando o sistema computacional (LEE, 2008). Segundo Wang, Törngren e Onori (2105), CPS afeta positivamente a manufatura na forma de sistemas de produção ciber-físicos (CPPS) na automação e controle de processos, porém ainda há enormes potenciais de aplicação do CPS na fabricação e ainda à falta de entendimento comum do CPS no setor manufatureiro.

Em geral, o CPPS com as características de discretização e rede oferece vantagens em termos de eficiência de recursos, adaptabilidade e estabilidade em relação aos sistemas de manufatura convencionais, bem como fornece todas as informações necessárias para executar o processo de fabricação com precisão e tomar decisões estratégicas, visto que para os vários comportamentos dos processos de fabricação, é necessária uma informação consistente e coerente do mundo físico (TANG et al., 2018).

Segundo Law e McComas (1998), a modelagem de sistemas de manufatura para simulação é utilizada desde o início da década de 1960 e tornou-se uma das mais populares e poderosas ferramentas empregadas para analisar sistemas complexos de manufatura.

O presente artigo tem como objetivo propor e analisar, por meio da aplicação da ferramenta de Mapeamento de Fluxo de Valor e simulação, a adoção de Sistemas Ciber-Físicos no fluxo de uma linha de produção de bobina de filme laminado, considerada como produto mais representativo no volume de produção de uma empresa produtora de embalagens flexíveis. Ademais, esta pesquisa tem como propósito comparar dois cenários de uma linha de produção de bobina de filme laminado, sendo eles: (i) cenário do estado atual do fluxo produtivo e (ii) um cenário do estado futuro integrando Lean com Sistemas Ciber-Físicos Inteligentes. Vale ressaltar que este artigo é direcionado para a exploração e enriquecimento do conhecimento disponível sobre Análise Baseada em simulação.

\section{REFERENCIAL TEÓRICO}

\subsection{Análise baseada em Simulação}

A simulação computacional é um processo que objetiva expressar o comportamento de um sistema, quando submetido a mudanças em seus fatores 
estruturais ou em suas condições de contorno, por meio da experimentação de um modelo do sistema real estudado, feita por meio de softwares que permitem testar várias estratégias e cenários, proporcionando a comparação de diversas alternativas operacionais viabilizando possibilidades para uma melhor decisão futura (HARRELL, GHOSH e BOWDEN, 2011.; SMEW, YOUNG e GERAGHTY, 2013; DE SOUZA et al., 2014). Pode ser utilizada para criar ou testar processos, políticas, operações e suas possíveis reações a um baixo custo e sem afetar o sistema real (BANKS, 1998).

Os modelos de simulação podem ser caracterizados em: modelos estáticos, representados por equações; dinâmicos, correspondendo a equações que variam ao longo do tempo; modelos contínuos, em que o tempo é considerado de forma linear; e eventos discretos em que o tempo é separado em pedaços distintos (MOURTZIS et al., 2015).

A gestão eficiente de sistemas de manufatura requer métodos confiáveis para o planejamento de processos e para a alocação de recursos, programação de tarefas, máquinas e pessoal, bem como para previsão dos prazos de entrega de cada pedido. No que se refere à tecnologia de manufatura, a analise baseada em simulação se constitui como informação valiosa para a programação e controle dos processos de produção (SILVA, 2017).

Outro importante objetivo da simulação em sistemas de manufatura é quantificar o desempenho do sistema (SILVA, 2017). Banks et al. (2000) cita as medidas de desempenho mais comuns para simulação de sistemas de manufatura: Rendimento sob demanda média ou picos de demanda; Tempo de ciclo do sistema; Utilização de recursos, máquinas e mão-de-obra; Gargalos; Filas e atrasos; Estoque necessário; Mão-de-obra necessária; Eficácia de sistemas de planejamento; Eficácia de sistemas de controle.

Robinson (2008) lista uma série de vantagens do uso da simulação na análise de operações de sistemas, incluindo menores custos e tempo de experimentos em comparação com sistemas reais, total controle das condições, modelagem da variabilidade, além de ser possível simular sistemas que ainda não existem no mundo real.

De acordo com Silva (2017), a análise baseada em simulação tem crescido nos últimos anos, sendo o aumento da capacidade computacional um dos fatores que contribuíram para a viabilização do uso dessa técnica, uma vez que a estimativa do 
valor da função-objetivo é "computacionalmente caro" em função do uso de um modelo de simulação.

\subsection{Mapeamento de Fluxo de Valor (MFV)}

MFV é a representação visual, com fluxo de material e informação de cada processo, utilizado pela Toyota. Rother formalizou a ferramenta após ter percebido o grande potencial que a mesma oferecia (ROTHER e SHOOK, 2003; SILVA, 2013; BONFANTE, 2016).

A ferramenta tem a capacidade de identificar desperdícios, para assim utilizar processos da Abordagem Lean o qual consiga eliminá-los (STADNICKA e ANTONELLI, 2015). Considerado um mapa de processo, o MFV é inserido para ser capaz de implementar táticas de gestão da empresa no nível operacional, pois é descrito como uma técnica utilizada para o diagnóstico, implementação e manutenção de uma abordagem enxuta, por meio da identificação e criação de valor (ROTHER e SHOOK, 2003; DAL FORNO et al., 2014; BONAMIGO, PAIMELL e ARBUGERI, 2017).

A finalidade é que o fluxo de valor seja analisado como um todo, e melhorias isoladas sejam evitadas, pois estas não refletem em redução de custo, nem melhorias para o cliente na qualidade e no serviço, nem benefício para o fornecedor. Entendese por fluxo de valor, o conjunto de ações necessárias para a produção de um produto, desde o consumidor até o fornecedor (ROTHER e SHOOK, 2003, BONFANTE, 2016).

O uso dessa ferramenta permite uma nova visão sobre o seu fluxo de valor, ao permitir um esboço de um fluxo de valor que seja considerado o mais ideal possível, com intuito de agregar o máximo de valor por meio da eliminação ou redução dos desperdícios, consequentemente, facilitando a implementação da Abordagem Lean (KAPPES, 2011; SILVA, 2013).

Em alguns casos, o desperdício é inevitável. Entretanto, o propósito do MFV é proporcionar a visualização de tais casos e avaliar se há a possibilidade de se reduzir ou eliminar o desperdício (BROWN, AMUNDSON e BADURDEEN, 2014).

Uma maneira de se gerenciar as complexidades do sistema de produção ao realizar o mapeamento é limitar o mapa apenas a elementos chave do fluxo (ROTHER e SHOOK, 2003). 
O mapeamento do fluxo de valor deve seguir, segundo Rother e Shook (2003), as etapas: (i) Escolha da família de produtos; (ii) Desenho o estado atual: Mapear o estado atual a partir de informações coletadas no chão da fábrica, isto é, representação visual da situação em que o processo se encontra e servirá de base para as propostas do desenho do estado futuro, com a utilização de conceitos e técnicas enxutas; (iii) Desenho o estado futuro: Mapear o estado futuro, o que é feito a partir de melhorias de processo a serem implementadas, devendo incluir o fluxo de materiais e informações idealizados pela empresa; e (iv) Plano de trabalho e implementação: Preparar um plano que descreva quais as melhorias que devem ser realizadas (SILVA, 2013; BONFANTE, 2016).

Abdulmalek e Rajgopal (2007) afirmam que o projeto de um estado futuro no MFV frequentemente está baseado na "crença", com base na experiência de outras empresas, de que o desempenho vai melhorar. Porém, essa justificativa não é suficientemente convincente para muitos gerentes, que precisam de evidências quantitativas que realmente comprovem a viabilidade do estado futuro projetado.

\subsubsection{MFV e Simulação}

A simulação tem sido aplicada em larga escala na busca dos melhores parâmetros de um processo de manufatura. Uma vez que a capacidade de visualização do que ocorre no sistema real, e a partir disto tomar decisões, realizar previsões e manutenções nos equipamentos. Neste âmbito, a simulação fornece aos tomadores de decisão uma visão sistêmica do processo (CORREA, MELLO e PEREIRA, 2015).

Para auxiliar uma organização a inserir técnicas enxutas e aprimoramento contínuo, uma ferramenta complementar para o mapeamento do fluxo de valor é necessária para quantificar os ganhos durante todas as etapas do planejamento. A simulação neste caso permite enxergar os detalhes, necessidades de recursos e estatísticas de desempenho, mantendo-se ainda flexível para diversas modificações pré e pós MFV da organização (DONATELLI e HARRIS, 2004).

A simulação computacional pode ser usada para reduzir a incerteza e criar exibições dinâmicas da situação futura do MFV, além de gerar mapas alternativos de 
acordo com diferentes cenários projetados. Isso permite aferir o retorno do uso do MFV e seu impacto sobre o sistema total (SOLDING e GULLANDER, 2009).

De acordo com Lima et al. (2016), o sucesso na utilização conjunta do MFV com a simulação de eventos discretos é confirmado por diversos exemplos encontrados na literatura, aplicados em diferentes contextos e com diferentes objetivos.

De acordo com McDonald, Van Aken e Rentes (2002), a incorporação da simulação permite predizer dinamicamente indicadores de desempenho do processo como níveis de estoque e tempos de atravessamento.

As vantagens da utilização conjunta do MFV com a simulação também são reconhecidas por Gurumurthy e Kodali (2011) e Oliveira, Corrêa e Nunes (2014), que propuseram modelos de simulação dentro do MFV tradicional. Segundo Lima et al. (2016) argumenta que os esforços para a integração entre simulação e MFV ajudam a preencher as lacunas deixadas pelo MFV original.

A utilização de MFV e simulação facilitam a implementação da manufatura enxuta, da mesma maneira que facilita a tomada de decisão dos gestores pelos melhores cenários (SHARARAH, EL-KILANY e EL-SAYED, 2010; SILVA, 2013).

\subsection{Sistemas Ciber-Físicos (CPS)}

O termo Sistemas Ciber-Físicos (CPS), foi cunhado nos Estados Unidos em 2006, com a compreensão da crescente importância das interações entre os sistemas de computação interconectados e o mundo físico (LEE, 2006; WANG, TÖRNGREN, E ONORI, 2105).

Define-se Sistemas Ciber-Físicos como tecnologia de gerenciamento de sistemas interconectados pelos seus recursos físicos e sua capacidade computacional que asseguram a comunicação entre humanos, máquinas e produtos similares, além autocontrolar certas atividades conforme eles adquirem e processam dados (BRETTEL et al., 2014; LEE et al., 2015; SILVA, 2017).

Segundo Wang, Törngren e Onori (2105), CPS é a integração dos processos computacionais e físicos, onde os computadores monitoram e controlam os processos físicos, recebendo e enviando feedback respectivamente. Portanto, a computação e a comunicação são utilizadas para adicionar novas competências aos sistemas físicos. 
Lee et al. (2015) afirma que a aplicação de CPS na produção, logística e serviços nas práticas industriais atual promoverá uma Indústria 4.0 com um significante potencial econômico. Atualmente, Indústria 4.0 é um termo popular para descrever as mudanças eminentes no cenário industrial, particularmente na indústria de produção e manufatura dos países desenvolvidos. Mas o termo ainda é usado em diferentes contextos e necessita de uma definição explícita (BRETTEL et al., 2014).

\subsubsection{Simulação Integrada Lean e CPS}

O desenvolvimento e a integração da automação digital da produção por meio de eletrônicos, tecnologia da informação (TI) e robôs industriais, levaram a sistemas de manufatura, atualmente denominados como CPS, que permitem uma produção altamente customizada e em massa (KAGERMANN et al., 2013; TORTORELLA et al., 2018).

Em contrapartida, Lean é uma abordagem amplamente disseminada que visa reduzir o desperdício e melhorar a produtividade e a qualidade de acordo com os requisitos dos clientes (WOMACK et al., 2007). Para Ritzman e Krajewski (2004), esta abordagem concentra-se em estratégias de operações, processos, tecnologia, qualidade, capacidade, arranjo físico, cadeias de suprimento, estoque e planejamento de recursos. Os sistemas de produção enxuta agrupam tudo para criar processos eficientes. Eles são conhecidos por muitas designações diferentes, que incluem estoque zero, fabricação sincronizada, produção sem estoque, material conforme o necessário e fabricação de fluxo contínuo, cada um com suas diferenças operacionais.

Sibatrova e Vishnevskiy (2016) afirmam que a Indústria 4.0 impactará na Abordagem Lean, permitindo superar as barreiras atualmente existentes e trazendo novos desafios para uma implementação bem-sucedida.

A recente integração entre as práticas da Abordagem Lean e as tecnologias da Indústria 4.0 foi intitulada como Automação Enxuta, e visa maior flexibilidade e fluxos de informações mais curtos para atender às futuras demandas do mercado (KOLBERG e ZÜEHLKE, 2015; TORTORELLA et al., 2018).

Com a indústria 4.0, empresas, departamentos e funções se tornarão mais coesas, realizando uma automatização da cadeia de valor. Ademais, a Industria 4.0 gerará: Capacidade de interação; Escalabilidade, adicionando ou retirando o número 
de recursos sem interromper as operações; Agilidade através da adaptabilidade e reconfiguração; Tolerância à falha e recuperação dela; Diversidade nos requisitos do produto; Produtos customizados; Lotes pequenos de produção, entre outros. Todos estes pontos são propósitos fundamentais da Abordagem Lean (JAMMES e SMIT, 2005).

No entanto, as abordagens atuais necessitam ser ajustadas às necessidades individuais. Evidências encontradas na literatura indicam que a compreensão dessa associação de Lean e Indústria 4.0, bem como seu impacto no desempenho operacional ainda precisam ser melhor explorados (EROL, SCHUMACHER e SIHN, 2016; SANDERS, ELANGESWARAN e WULFSBERG, 2016).

Murayama, Reston Filho e Cardoso (2017) indicam que o MFV está intrinsicamente atrelado à Industria 4.0 por permitir a virtualização do produto e serviços, produção, fornecimento, consumo e negócios. O MFV é a realização completa da virtualização de uma empresa e permite tornar seus processos físicos em digitais, levando a possibilidade de simular contextos e resultados. Dentro do contexto Industria 4.0, o MFV deixa de operar no eixo horizontal (fluxo de informação - fluxo de material) para o eixo vertical amarrando a operação fabril com as estratégias da empresa passando pelos processos de desenvolvimento de produto e de Serviços.

\section{PROCEDIMENTOS METODOLÓGICOS}

Esta pesquisa segue a metodologia de estudo de caso. Segundo Mascarenhas (2012), o estudo de caso é uma pesquisa bem detalhada sobre um ou poucos objetos, em que seu objetivo é entender o conjunto geralmente diversificado de dados do caso concreto e, desta forma, retratá-lo com maior profundidade. Isto significa que, por meio deste método de pesquisa, é possível entender muito bem o contexto analisado.

O esquema representativo do desenho da pesquisa apresenta em detalhe 0 escopo deste trabalho, conforme a Figura 1, que mostra como a mesma foi desenvolvida, hierarquizando seu passo a passo bem como os métodos que serão adotados. 
Figura 1 - Metodologia de condução da pesquisa

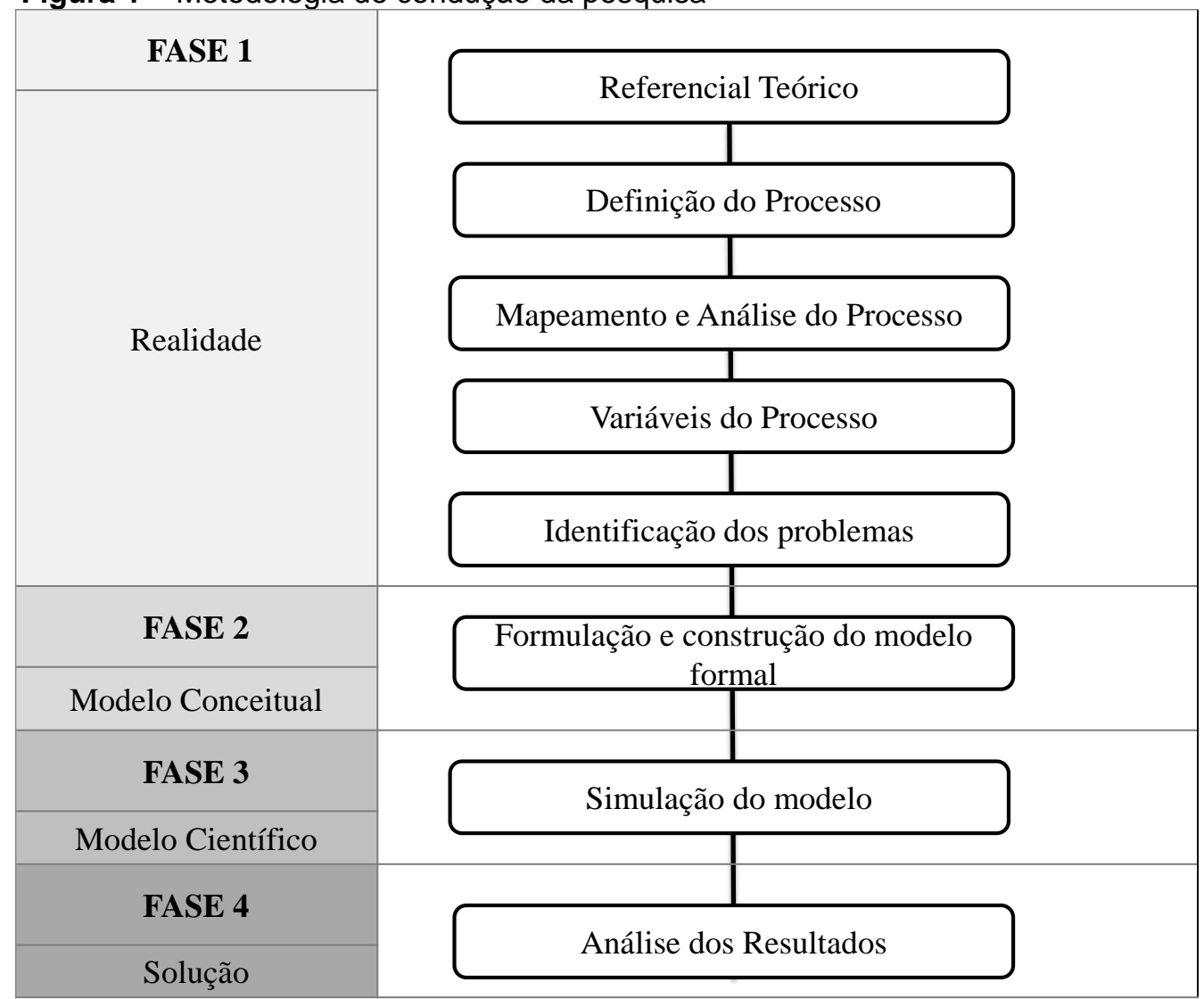

Fonte: Adaptado de Mitroff et al. (1974)

A metodologia desta pesquisa, apresentado na Figura 2, se baseou em uma adaptação do modelo proposto por Mitroff et al. (1974), em que foi dividida em quatro fases: Realidade, Modelo Conceitual, Modelo Científico e Solução.

A primeira fase correspondeu a construção do referencial teórico por meio de uma busca exploratória sobre os temas relevantes à pesquisa. Também, caracterizouse o processo a ser estudado, bem como foi realizado o seu mapeamento e análise elaborados a partir da interpretação da realidade, problema ou situação que está sendo estudado. A análise do processo permitiu a identificação dos seus problemas.

Em seguida, na segunda fase, elaborou-se o modelo conceitual, representado pelos Mapas do Estado Atual e Futuro. Depois, na terceira fase, estes modelos foram transformados em modelos de simulação ou computacionais, com auxílio de um software.

Mitroff et al. (1974) destaca que se necessário pode haver a realimentação do modelo conceitual e o ciclo pode continuar a partir deste modelo conceitual alterado. 
Ao final do método, as soluções obtidas da simulação podem ser aplicadas na realidade.

A simulação de eventos discretos tem seu procedimento classificado como experimental e laboratorial, pois explora o objeto de estudo em um ambiente controlado. O trabalho é realizado utilizando um método indutivo com abordagem de documentação direta, quantitativa e qualitativa. A partir da análise de dados quantitativos específicos de alguns casos, obtêm-se conclusões mais amplas para todos os cenários (GIL, 2010).

\section{MODELO CONCEITUAL}

O modelo conceitual é uma representação matemática, lógica ou gráfica do problema de um determinado estudo. Pode ser considerado como a abstração de modelos ou sistemas reais (SARGENT, 2013; FURIAN et al., 2015). Para a simulação, o modelo conceitual representa um detalhamento do processo que será modelado e simulado, contendo os objetivos, entradas, saídas, suposições e as simplificações envolvidas na simulação, porém sem a utilização de softwares (ROBINSON, 2008).

Segundo Law (1991), este passo de concepção do modelo conceitual é o aspecto mais importante de um estudo de simulação. Além disso, facilita a realização das demais etapas do projeto de simulação. Segundo Silva et al. (2014), a modelagem conceitual permite uma implementação mais fácil do modelo computacional. E, segundo Banks et al. (2000), há uma interação contínua entre a construção do modelo conceitual e a identificação dos dados necessários.

De acordo com Hernandez-Matias et al. (2008), existem várias técnicas de modelagem de processos, isto é, não há um único método de modelagem conceitual que pode modelar completamente um sistema complexo de manufatura. A Figura 2 a seguir apresenta a estrutura conceitual genérica da pesquisa, que posteriormente, será testada em um caso específico por meio do uso de dados empíricos e abordagem de simulação. 
Figura 2 - Modelo Conceitual

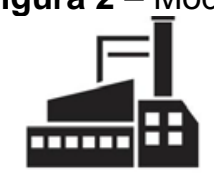

Indústria

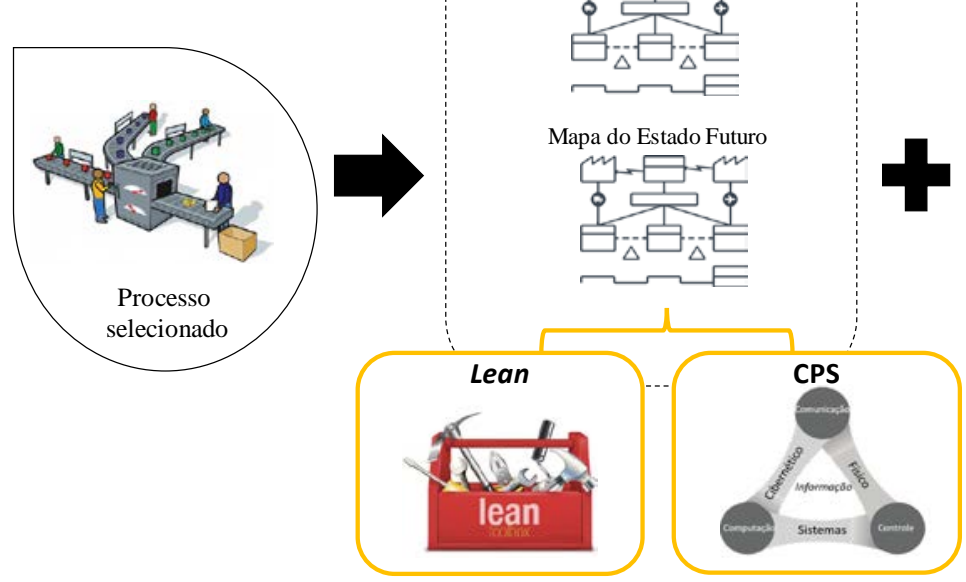

Análise baseada em

Simulação

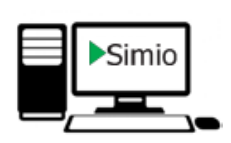

Simulação integrada Lean e CPS

Fonte: Elaborada pelos autores

O mapeamento de processo pode ser utilizado como forma de descrever a lógica e determinar os pontos de decisão, antes mesmo do modelo computacional (GREASLEY, 2006; KUMAR e PHROMMATHED, 2006). Para esta pesquisa, a técnica escolhida foi a ferramenta Mapeamento de Fluxo de Valor, na qual obteve-se os mapas de estado atual da linha de produção de bobinas laminadas e elaborou-se o mapa de estado futuro ao promover a integração de ferramentas Lean e CPS. Após confecção dos mapas, usa-se um software de simulação.

\section{CASO TESTE}

\subsection{Ambiente de pesquisa}

A empresa estudada produz embalagens plásticas flexíveis para alimentos, higiene, limpeza, indústria, lojas de departamentos, material promocional, pet food, saúde e supermercados. São processadas 130 toneladas de produto por mês, sendo o principal material processado o polietileno de baixa densidade. Os principais clientes desta empresa se situam no Rio Grande do Sul e seus principais fornecedores são de São Paulo e do Paraná.

Os processos produtivos da empresa compreendem: extrusão, impressão, laminação, rebobinamento, corte e soldagem. Estes processos estão organizados em 
forma de leiaute funcional, representados na Figura 3, em que processos similares são localizados próximos uns dos outros (SLACK, CHAMBERS e JOHNSTON, 2009).

Figura 3 - Layout da empresa

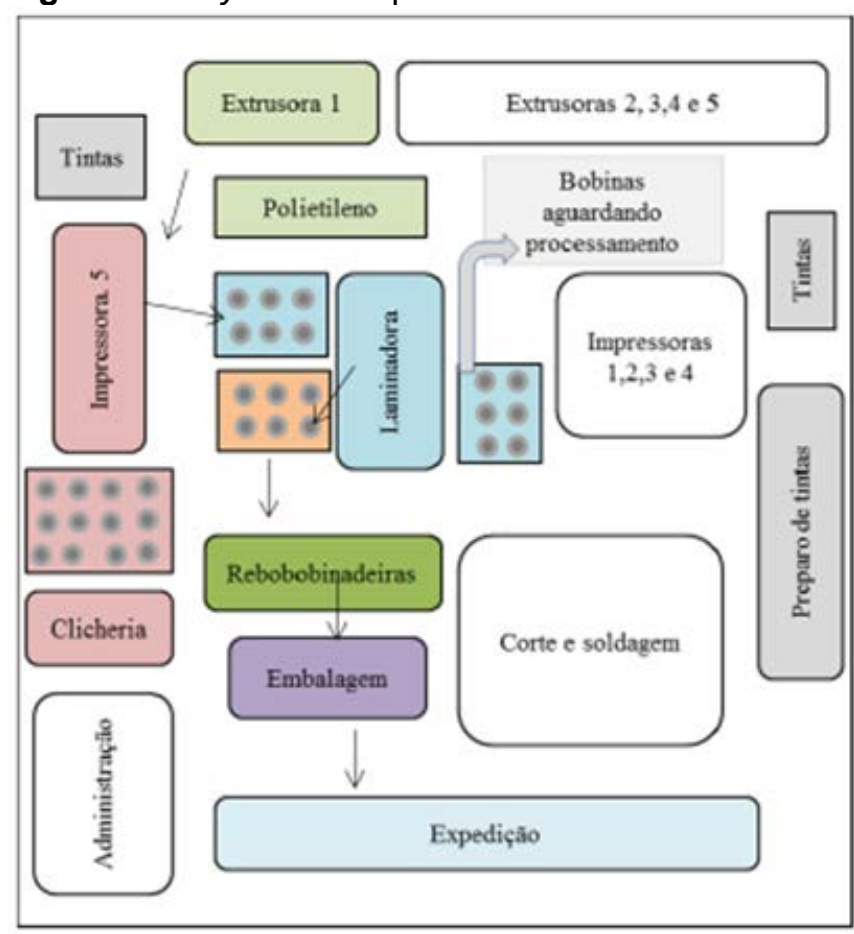

Fonte: Bonfante (2016)

Segundo Rother e Shook (2003), uma família de produtos é caracterizada por máquinas similares nos seus processos e por etapas comuns de processamento. $A$ família de produtos que serve como foco central para esta pesquisa é a de laminados, mais especificamente o processo de fabricação de bobinas de filme laminado para embalagens de $1 \mathrm{~kg}$ de alimentos. Esta linha é a mais representativa no volume de produção da empresa, correspondendo em média 15 toneladas ao mês. Os consumidores destas bobinas de filme são beneficiadores de alimentos, principalmente de arroz e feijão.

O processo produtivo de bobinas de filme laminado para embalagens de $1 \mathrm{~kg}$ se inicia com o processo de extrusão e, em seguida, a bobina de filme passa para o processo de impressão. As etapas seguintes são a laminação e a cura, seguidas da etapa de rebobinamento. Finalmente o produto passa pelas etapas de embalagem e expedição. Seguindo este mesmo fluxo, e utilizando a mesma estrutura, outros produtos também são produzidos. A Figura 4 apresenta o fluxo atual do processo, que constitui o limite de estudo deste trabalho. 
Figura 4 - Fluxo da produção de bobinas de filme laminado

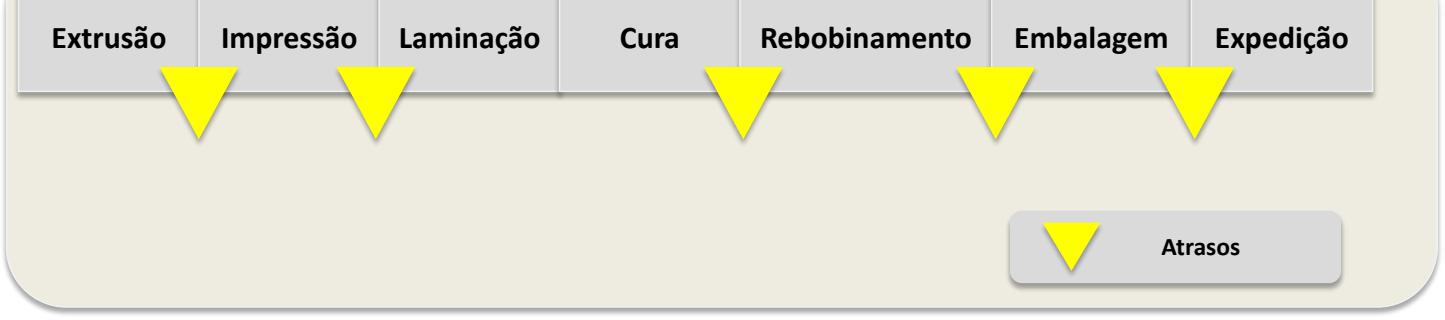

Fonte: Elaborado pelos autores

\subsection{Coleta de Dados}

A coleta de informações ocorreu in loco, por meio de observações e entrevistas com os funcionários envolvidos (mão-de-obra direta) e pessoal da direção. O grupo envolvido na aplicação da ferramenta foi composto pelo gerente de produção, por um supervisor de produção e pela responsável pela qualidade.

Os dados resultantes da monitoração do sistema real possibilitaram a construção do Mapa do Estado Atual e as projeções sobre um sistema a ser desenvolvido foram apresentadas no Mapa do Estado Futuro.

Cabe destacar que os dados para a construção do mapa do estado atual foram coletados no período de um mês (BONFANTE, 2016). Tais dados foram submetidos ao Critério de Chauvenet, a fim de realizar a exclusão de valores atípicos, denominados outliers (TAYLOR, 2012).

\subsection{Mapa do Estado Atual}

Conforme Venturini (2012), deve-se realizar uma análise prévia do processo produtivo e identificar os problemas existentes. Dessa forma, fez-se uma análise de todo o fluxo de valor a fim de compreendê-lo, inclusive a sequência do processo. Para o desenho do estado atual, após a família de bobinas laminadas para embalagens de $1 \mathrm{~kg}$ ter sido selecionada, as informações foram coletadas a partir da expedição final (expedição de peças acabadas) até a matéria prima recebida.

O objetivo desta etapa foi identificar o tempo médio de atravessamento para cada unidade de produto entregue ao cliente, assim como identificar os pontos de espera (BONFANTE, 2016). A empresa trabalha com turnos diferenciados em cada 
etapa do processo. Os horários de trabalho, o número de operadores e o tempo disponível durante um dia são apresentados na Tabela 1.

Tabela 1 - Distribuição de turnos e operários por processo

\begin{tabular}{|c|c|c|c|}
\hline Etapa & \multicolumn{2}{|c|}{ Horário e número de operadores } & Tempo disponível \\
\hline \multirow{2}{*}{ Extrusão } & 13:00-21:00 & 21:00-05:00 & \multirow{2}{*}{$1440 \mathrm{~min}$} \\
\hline & 2 operadores & 2 operadores & \\
\hline \multirow{2}{*}{ Impressão } & $22: 20-07: 30$ & $07: 30-17: 30$ & \multirow{2}{*}{$1200 \mathrm{~min}$} \\
\hline & 2 operadores & 2 operadores & \\
\hline \multirow{3}{*}{ Laminação } & $22: 20-07: 30$ & $07: 30-17: 30$ & \multirow{3}{*}{1006 min } \\
\hline & 1 operador & 1 operador & \\
\hline & (intervalo 72 min) & (intervalo $72 \mathrm{~min}$ ) & \\
\hline \multirow{2}{*}{ Cura } & \multirow{2}{*}{\multicolumn{2}{|c|}{$\begin{array}{c}07: 30-17: 30 \\
1 \text { operador (intervalo } 72 \mathrm{~min} \text { ) } \\
\end{array}$}} & \multirow{2}{*}{$528 \mathrm{~min}$} \\
\hline & & & \\
\hline \multirow{2}{*}{ Rebobinamento } & \multirow{2}{*}{\multicolumn{2}{|c|}{$\begin{array}{c}07: 30-17: 30 \\
2 \text { operadores (intervalo } 72 \mathrm{~min} \text { ) }\end{array}$}} & \multirow{2}{*}{$528 \mathrm{~min}$} \\
\hline & & & \\
\hline \multirow{2}{*}{ Embalagem } & \multicolumn{2}{|l|}{$07: 30-17: 30$} & \multirow{2}{*}{$528 \mathrm{~min}$} \\
\hline & 1 operador (intervalo & $72 \mathrm{~min})$ & \\
\hline \multirow{2}{*}{ Expedição } & $07: 30-17: 30$ & & \multirow{2}{*}{$528 \min$} \\
\hline & 1 operador (intervalo & $72 \mathrm{~min})$ & \\
\hline
\end{tabular}

Fonte: Bonfante (2016)

Os processos produtivos da empresa trabalham de forma empurrada, isto é, o processo produz alguma coisa independentemente das necessidades reais do processo-cliente seguinte e "empurra para frente" (ROTHER e SHOOK, 2003).

A partir dos dados coletados determinou-se o tempo médio de processamento em cada etapa do processo para entregar um produto ao cliente, além dos tempos de espera entre os processos. A Figura 5 apresenta os tempos médios de processamento para as etapas que envolvem transformação de matéria-prima. 
Figura 5 - Tempo de processamento

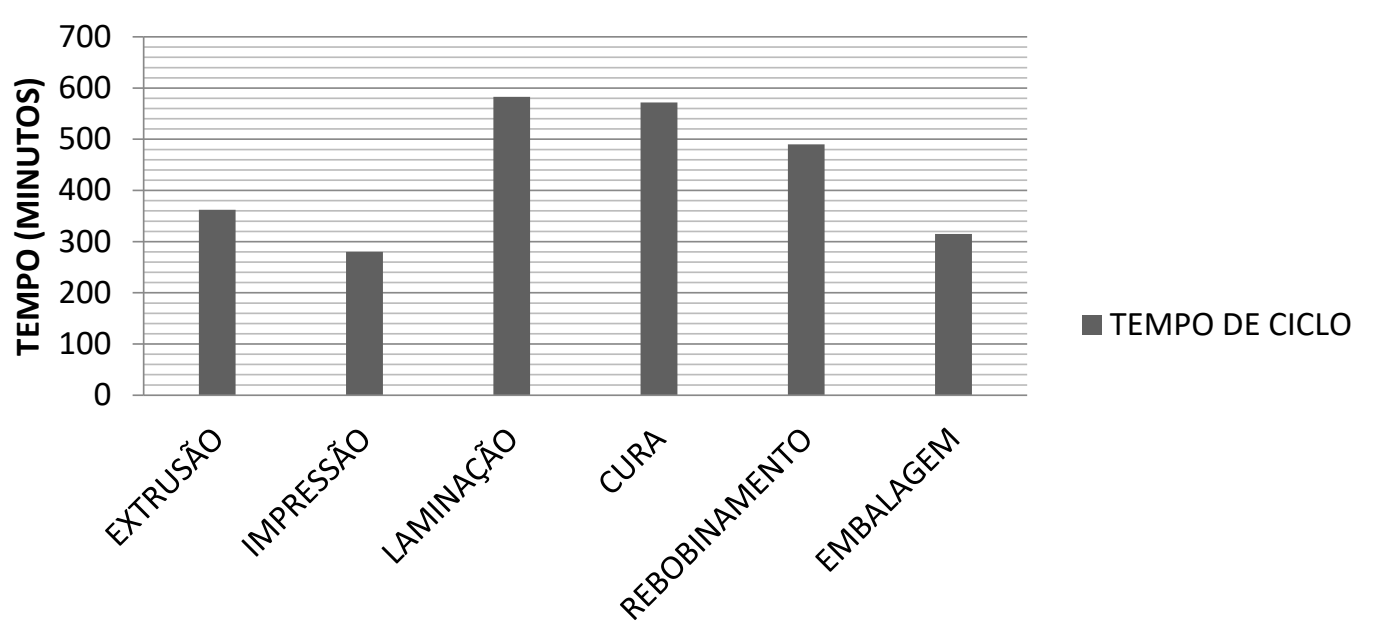

Fonte: Bonfante (2016)

A Figura 6 apresenta os tempos de setups das operações. Observa-se que o valor de setup da Impressão é o maior diante dos demais.

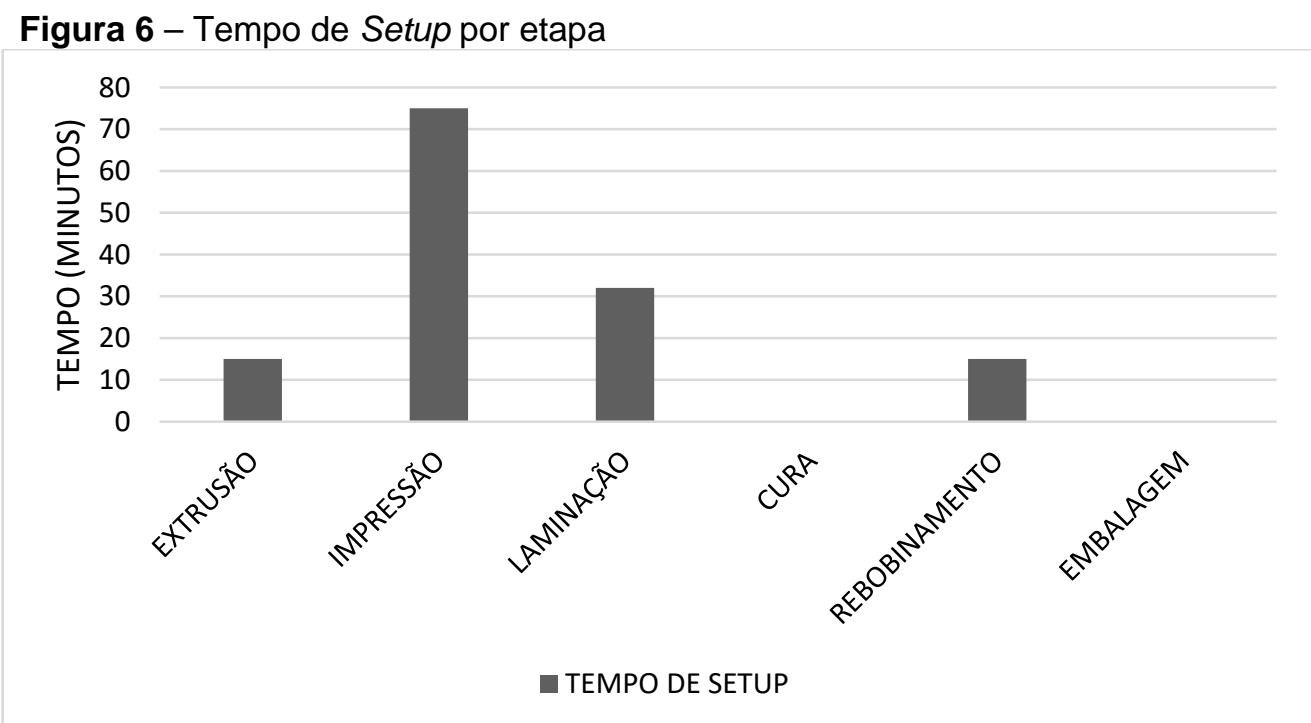

Fonte: Bonfante (2016)

A Figura 7 apresenta as quantidades de perda em relação ao total de material processado em cada etapa. A etapa de rebobinamento foi a que apresentou o maior valor de perda de matéria-prima, correspondendo a $6,3 \%$ da quantidade de filme processado nesta etapa, seguida da etapa de impressão, que apresentou 2,4\% de perdas e da etapa de extrusão, que apresentou 2,3\% de perdas. A etapa de laminação teve $1,2 \%$ de perda e as etapas de cura e embalagem não apresentaram perdas. 
Figura 7 - Perdas em cada etapa

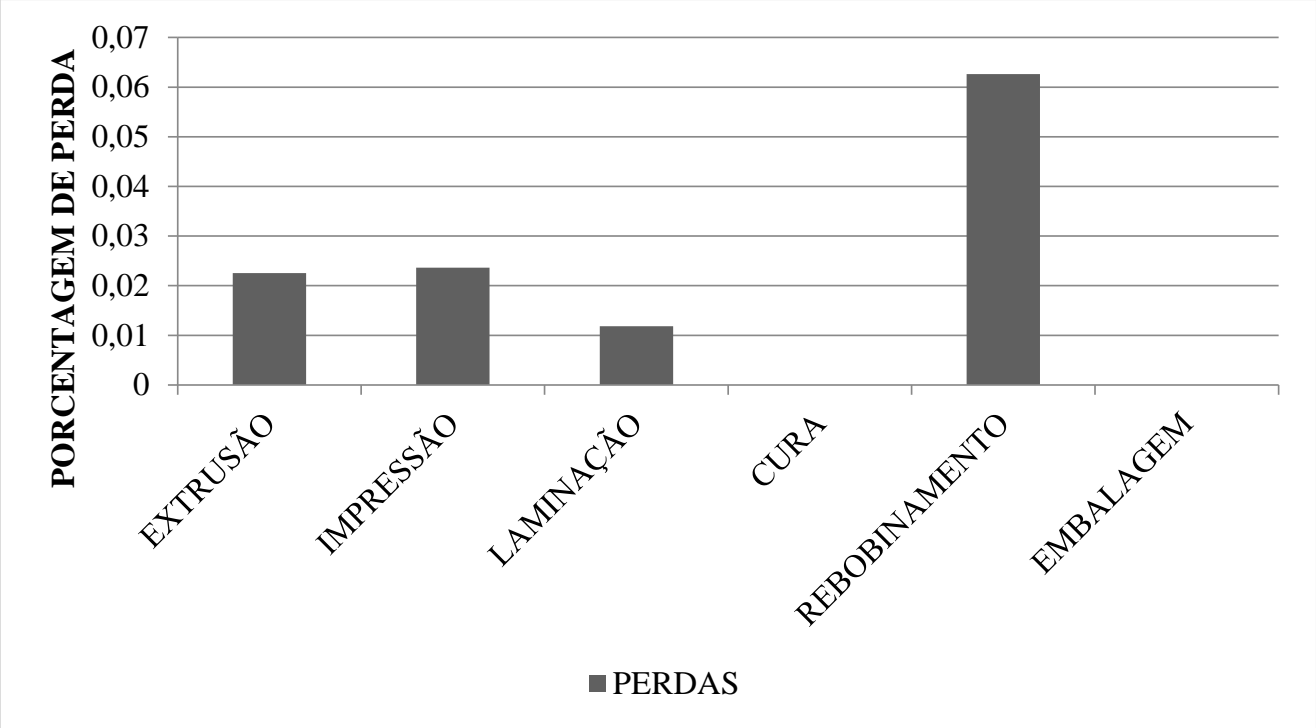

Fonte: Bonfante (2016)

Elaborou-se o fluxo de material na parte de baixo do mapa do Estado Atual, representado na Figura 8. Este fluxo segue a sequência das etapas do processo, sendo que cada etapa é representada por uma caixa de processo. Esta contém as informações básicas do processo, tais como: número de operadores, tempo de ciclo, tempo de troca, disponibilidade real da máquina, tamanho dos lotes de produção, tempo de trabalho, taxa de perdas. Abaixo de cada caixa de processo deve-se representar uma linha do tempo, com as informações de tempos de cada etapa produtiva. Os estoques entre os processos também estão representados. Ao final, tem-se o tempo de atravessamento (lead time) do processo mapeado, assim como o tempo de processamento. 
Figura 8 - Mapa do Estado Atual

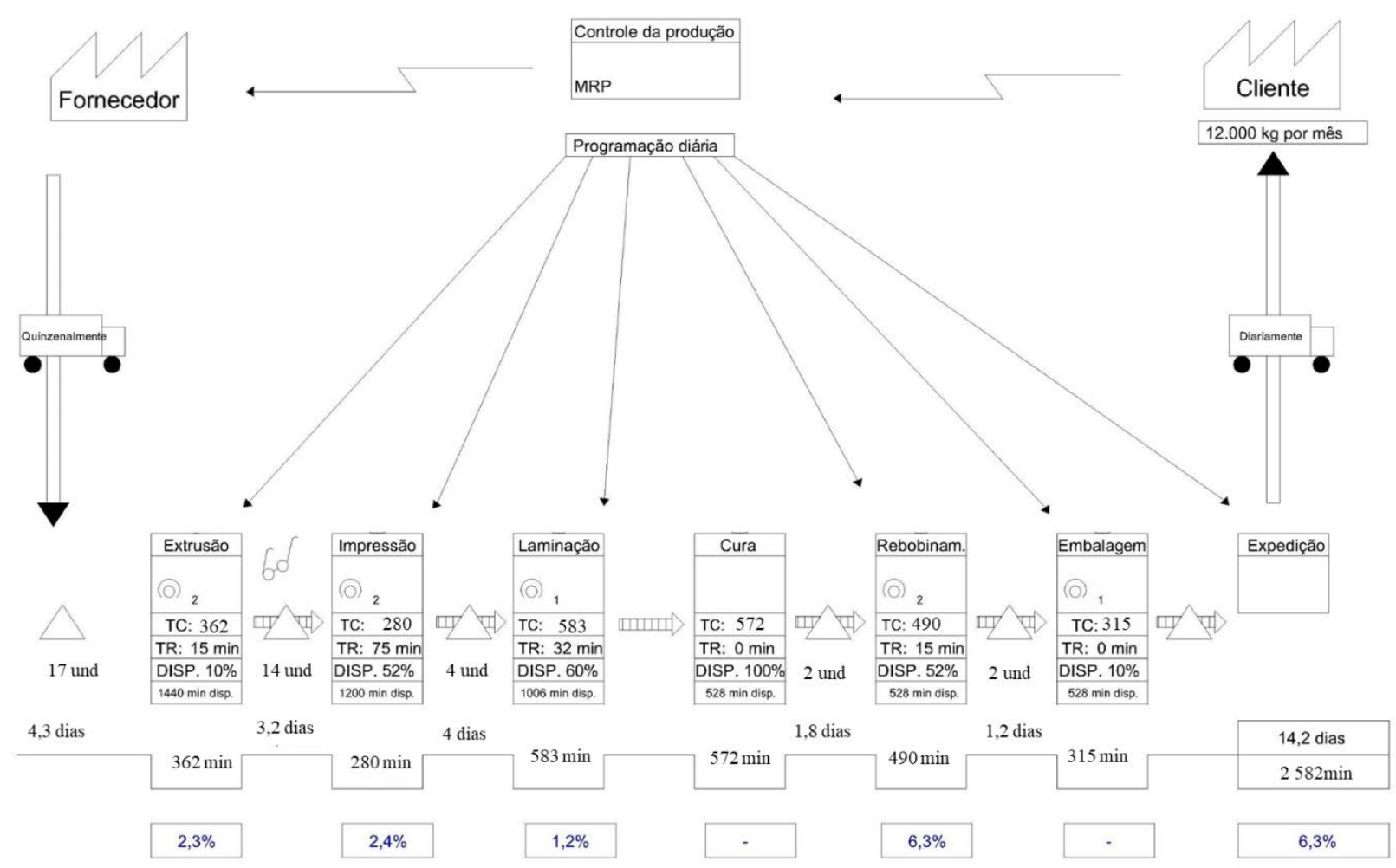

Fonte: Bonfante (2016)

\subsubsection{Avaliação do Mapa do Estado Atual}

A avaliação do mapa do estado atual teve como objetivo identificar os principais pontos de desperdício no processo produtivo, conforme listado abaixo:

- Elevado tempo de setup na etapa de impressão;

- Elevado tempo de setup na etapa de laminação;

- Maior tempo de processamento para a fabricação a partir de bobinas simples;

- Necessidade de conferência de estoque diário e emissão de ordens de fabricação no estoque de bobinas lisas;

- Elevadas perdas de materiais no processo, principalmente na etapa de rebobinamento, com 6,3\% de material descartado;

- Elevado estoque de bobinas lisas;

- Elevada variabilidade no tempo de cura;

- Elevado estoque de matéria prima (Polietileno Linear de Baixa Densidade e Polietilino Linear). 


\subsection{Mapa do Estado Futuro}

Seguindo a metodologia proposta por Rother e Shook (2003), com base na análise do mapa do estado atual, elaborou-se o mapa um estado futuro que reduzisse ou eliminasse os desperdícios encontrados, ao propor uma nova alternativa que vise um fluxo de valor enxuto que proporcionasse ao cliente uma bobina com alto valor agregado.

De acordo com Rother e Shook, (2003), busca-se implementar um processo capaz de fazer somente o que o processo seguinte necessita e quando necessita. Para tanto, todos os processos devem ser interconectados em um fluxo regular, que resulte no menor tempo de atravessamento, na mais alta qualidade e no menor custo.

No desenvolvimento do mapa do estado futuro, buscou-se projetar o estado almejado pela empresa, ou seja, o BSV (Blue Sky Vision) para o processo. Apresentado na Figura 9. Neste mapa, tem-se a diminuição de perdas, diminuição dos tempos de setup, implementação de supermercado, diminuição da variabilidade da etapa no tempo de cura, implementação de Kanban.

Também, introduz-se Sistemas Ciber-físicos a linha de produção estudada, por meio do cadenciamento de floor shop, na qual o status de uma máquina influencia na anterior. Quando sair um produto de determinada máquina, acionar a produção da outra. Em caso de perda, acionar uma entrada.

Para isso, a empresa busca diminuir o estoque de matéria-prima aguardando processamento, possibilitando reduzir o tempo de atravessamento (lead time). A criação de um supermercado no estoque de bobinas lisas e um no estoque de polietileno também é um objetivo para a situação futura almejada e, desta forma, elimina-se a necessidade de conferência diária de estoque. Além disso, a produção das próximas etapas será regida pelo método FIFO (First in First Out). Os tempos de setup da etapa de impressão e laminação devem levar no máximo 30 minutos e 20 minutos respectivamente (BONFANTE, 2016). 
Figura 9 - Mapa do Estado Futuro

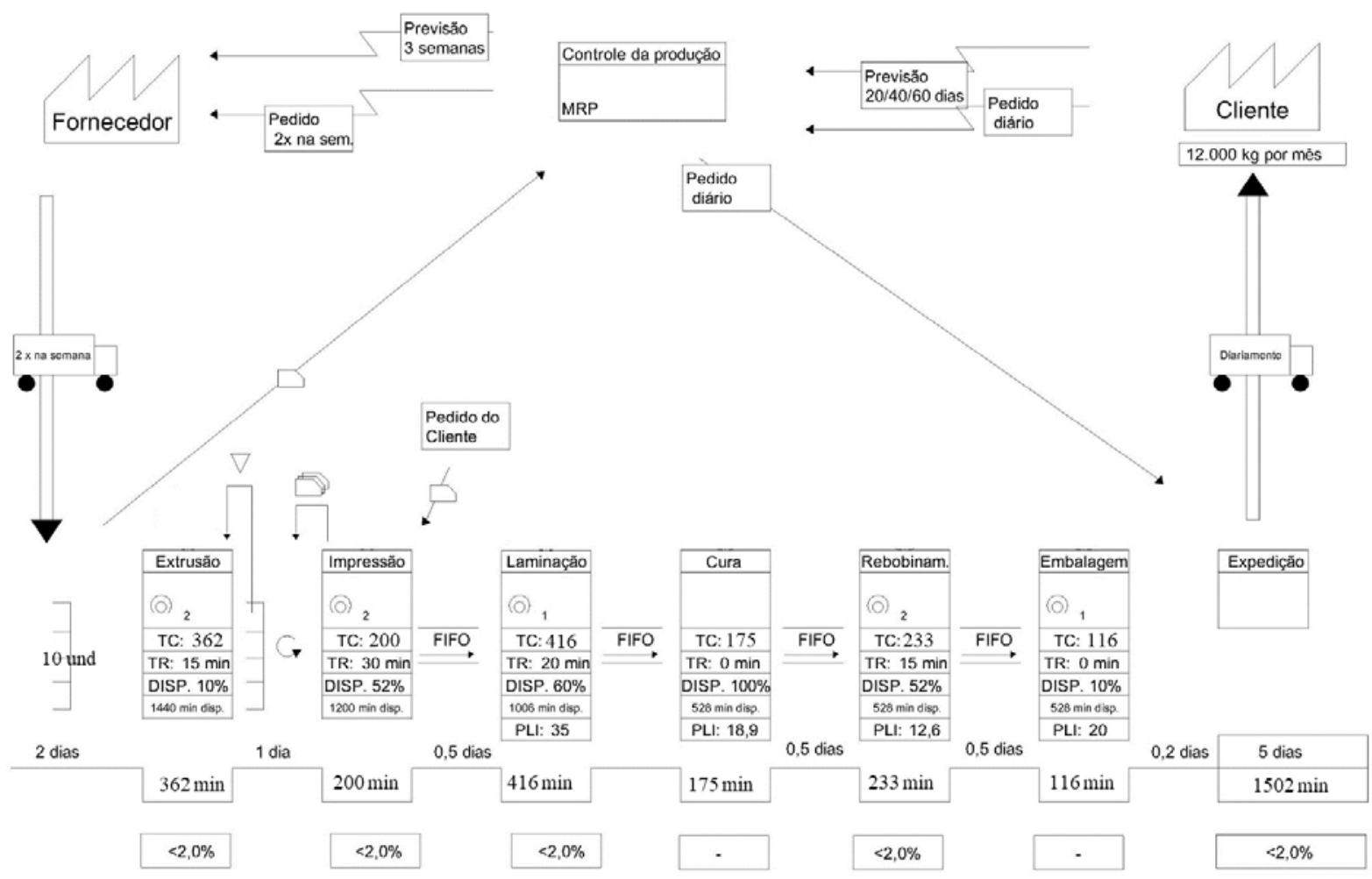

Fonte: Bonfante (2016)

\subsection{Modelo de Simulação}

Um modelo de simulação é uma réplica reduzida ou simplificação de um conjunto de variáveis de um sistema real (MIHRAN e MIHRAM, 1974; BANKS et al., 2000).

De acordo com Ruiz et al. (2014), os modelos de simulação têm o potencial de prover mais do que a informação que é requerida e reduz os riscos no projeto, análises e a operação em sistemas complexos. Muitas das ferramentas de simulação são focadas para resolver problemas relacionados ao planejamento de manufatura. Existem ferramentas de simulação que oferecem optimizações para melhorar modelos estatísticos antes de simular o processo, e outras ferramentas de simulação que permitem usar e modificar o modelo.

O campo da manufatura permite a aplicação da simulação de cenários sendo uma ferramenta eficaz para validação de métodos e arquiteturas antes de serem aplicadas no chão de fábrica. Sistemas de tecnologia têm demostrado utilidade para sistemas de manufatura com a interação e a cooperação de cenários com agentes 
específicos para70 facilitar a simulação da manufatura em um caminho apropriado e flexível (KAIHARA et al., 2017).

A construção dos modelos de simulação desta pesquisa foi realizada utilizando

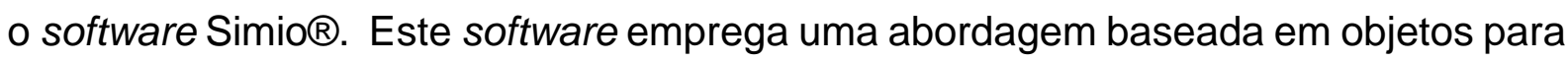
modelagem, onde modelos são construídos a partir da combinação de objetos que representam componentes físicos do sistema. Além disso, requer variáveis de entrada (tempo por setor, quantidade de erros por peça) para conseguir estruturar o funcionamento do processo e as variáveis de saída. Com estes parâmetros obtém-se um resultado do desempenho no sistema (PEGDEN e STURROCK, 2012).

\subsubsection{Cenários}

O cenário dos modelos representa a linha de bobinas laminadas de uma empresa de embalagens flexíveis, conforme mencionado anteriormente. As variáveis de entrada deste modelo são: número de operadores, tempo de ciclo, tempo de troca, disponibilidade real da máquina, tamanho dos lotes de produção, tempo de trabalho, taxa de perdas.

Com esta simulação, estimou-se verificar o comportamento da produtividade da linha com implementação de melhorias por meio da integração Lean e CPS a fim de mensurar os possíveis ganhos. Visto que esses sistemas são capazes de fornecer novos níveis de desempenho e eficiência graças a sofisticados códigos de controlecomputação (WOLF, 2009). Os cenários projetados nesta pesquisa e suas características estão apresentados na Quadro 1.

Quadro 1 - Cenários da pesquisa

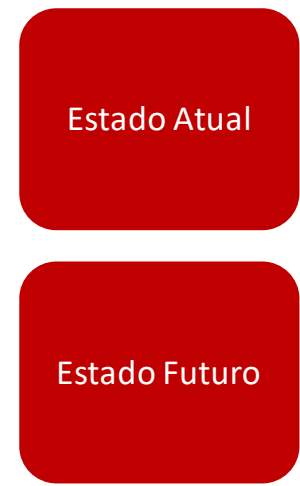

Elevado tempo de setup; Elevadas perdas de materiais no processo; estoque intermediário; elevada variabilidade no processo de cura, entre outros

Fonte: Elaborado pelos autores 
Para a representação do estado real, utiliza-se os dados coletados em conjunto com os processos. Assim o sistema modelado é testado até coincidir com os dados levantados. Desta forma os dados coletados são os inputs de informações para a elaboração do modelo no software de simulação.

A Figura 10 apresenta o modelo de simulação referente ao Mapa do Estado Atual da linha de bobinas laminadas, apresentado na Figura 9 da seção 4.1.1. Após a construção do modelo (posicionamento dos objetos e os caminhos disponíveis para o fluxo de material), foram adicionados os dados ao modelo.

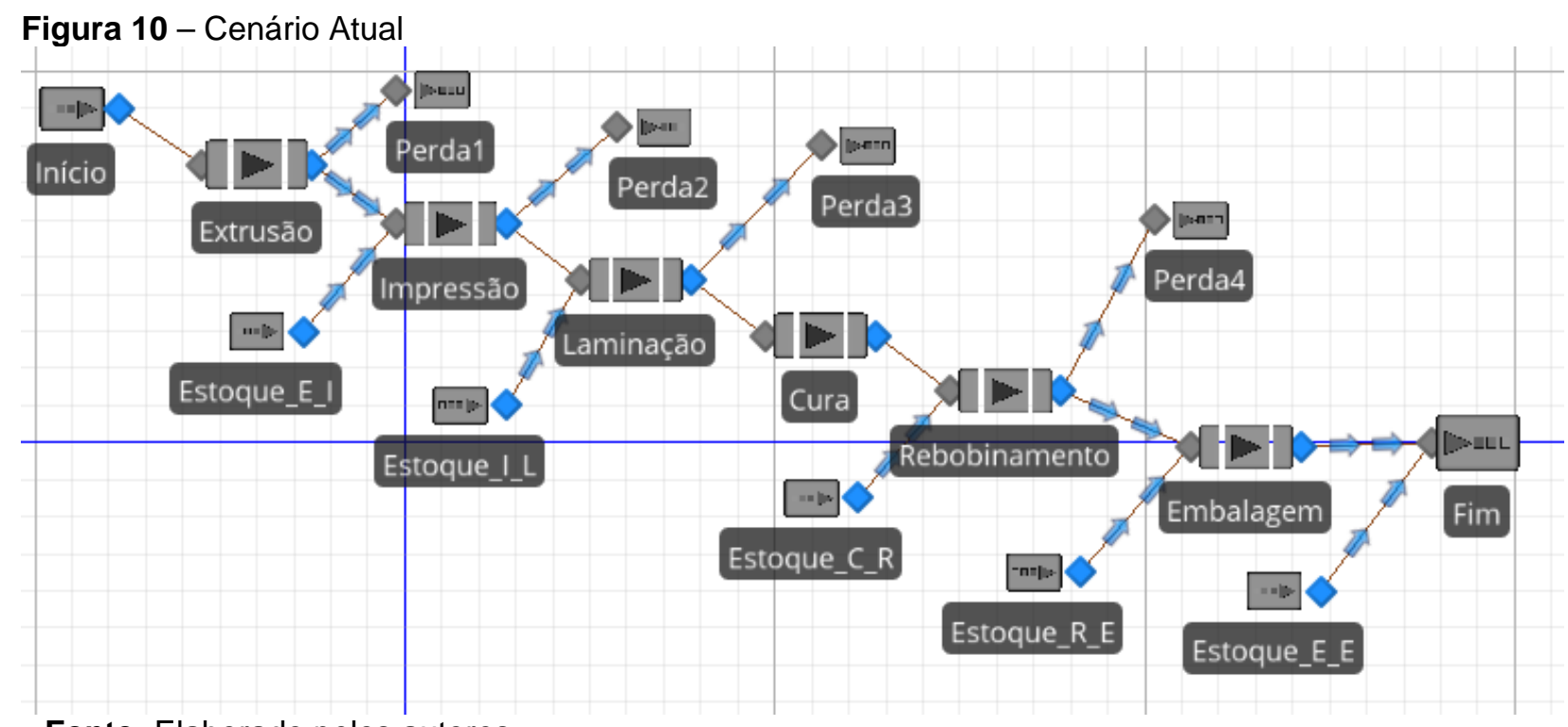

Fonte: Elaborado pelos autores

No segundo momento, constrói-se o modelo de simulação do Estado Futuro, apresentado na Figura 11, que promove a integração de Lean e CPS. Neste modelo, incluiu-se as melhorias descritas no tópico 4.1.2. As melhorias são pertencentes a Abordagem Lean e à Sistemas Inteligentes. Vale ressaltar que quando sair um produto de determinada máquina é acionada a produção da outra. 
Figura 11 - Cenário Futuro

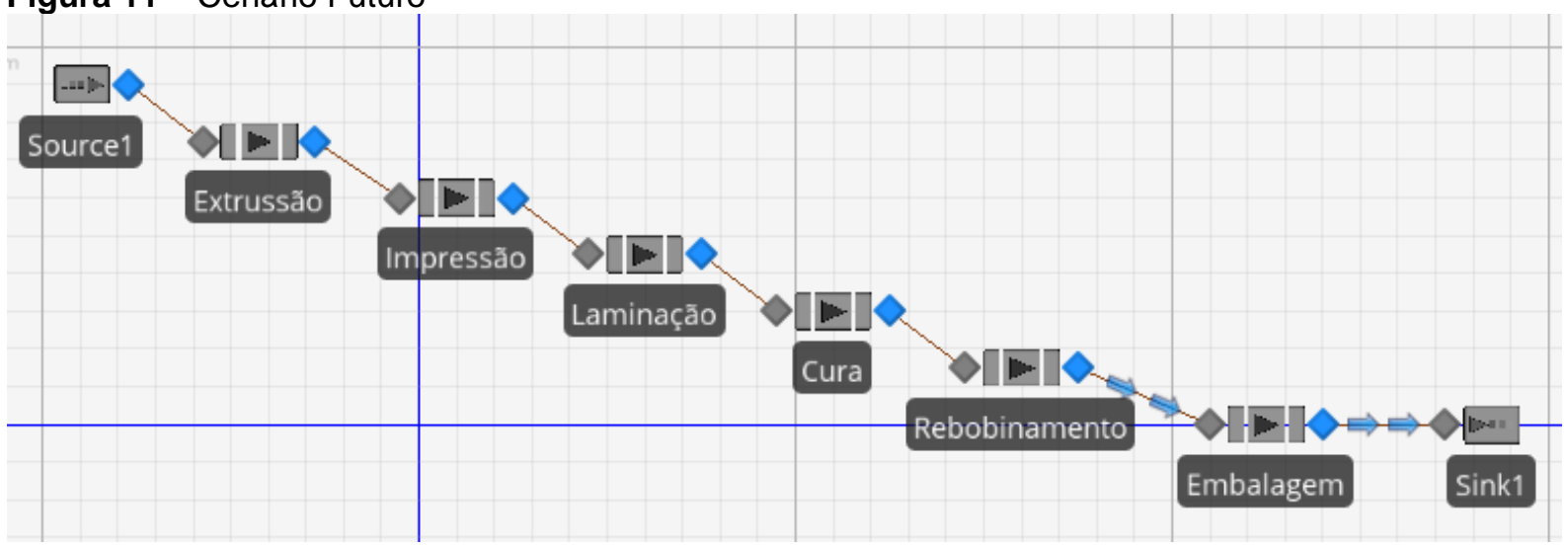

Fonte: Elaborado pelos autores

\subsubsection{Análise dos Resultados}

Comparando o cenário atual com futuro, houve aumento de significativo de produtividade. No cenário atual, 22 bobinas eram fabricadas em um período de 14 dias. No futuro, 22 bobinas são fabricadas em 5 dias.

Estes resultados apontam que a integração Lean com CPS ao longo da linha de produção de bobinas laminadas implica na melhoria deste processo e em um ganho na utilização dos recursos da indústria de embalagens. Observa-se ainda que não houve alterações no que diz respeito ao controle ou gerenciamento do processo. Apenas relacionado a automação na troca de informação em processos já existentes.

\section{CONSIDERAÇÕES FINAIS}

A principal função da simulação de processos é viabilizar o estudo de conceitos de alta complexidade de aplicação em sistemas reais (SILVA, 2017). Sendo assim, esta pesquisa teve como objetivo propor e analisar, por meio da aplicação da ferramenta de Mapeamento de Fluxo de Valor e simulação, a adoção de Sistemas Ciber-Físicos no fluxo de uma linha de produção de bobina de filme laminado.

Esta pesquisa demonstrou a importância atribuída ao modelamento conceitual, fase esta que antecede o modelo computacional em projetos de simulação. O uso do Mapeamento de Fluxo de Valor se mostrou adequado para a modelagem conceitual, permitindo o modelamento do caso prático demonstrado no artigo.

Com o MFV, foi possível realizar a identificação das necessidades de coleta de dados de forma fácil, listando tempos e probabilidades necessários, com base na 
afirmativa de Banks et al. (2000), de que há uma interação contínua entre a construção do modelo conceitual e a identificação dos dados necessários.

Utilizar o MFV é vantajoso pelo fato da ferramenta permitir a facilidade de visualização dos problemas que existem no processo produtivo a fim de elimina-los com base nos princípios da Abordagem Lean. Porém, uma das desvantagens de se utilizar somente o MFV é que as análises são feitas considerando-se dados predeterminados, ou seja, não possuem a característica aleatória. O uso da simulação em concomitância com o MFV é que esta fornece suporte a ferramenta na busca de contribuir para a redução das limitações do uso de somente o MFV (SILVA, 2013).

Ao propor um cenário futuro com a integração de Lean com CPS (Sistemas Ciber-físicos), esta pesquisa proporciona informações para a tomada de decisão ser a necessidade de interferir no sistema real por meio da análise baseada em simulação. Com estas informações, os gestores da empresa de embalagens podem compreender melhor os possíveis impactos gerados pela adoção das novas políticas. Além disso, a comparação da aplicação do procedimento em um caso real com a aplicação em sistemas teóricos e aleatórios aumenta a credibilidade de toda a contribuição teórica desenvolvida neste trabalho e no trabalho de autores que não conseguiram provar suas teorias, visto que muitos dos resultados convergem para o resultado esperado, ou para o senso comum.

\section{REFERÊNCIAS}

ABDULMALEK, F.A.; RAJGOPAL, J. Analyzing the benefits of Lean manufacturing and value stream mapping via simulation: a process sector case study. International Journal of Production Economics, v. 107, n. 1, p. 223-236, 2007. DOI:

http://dx.doi.org/doi:10.1016/j.ijpe.2006.09.009

BANKS, J. Handbook of simulation: principles, methodology, advances, applications and practice. New York: John Wiley \& Sons, Inc., 1998.

BANKS, J.; CARSON, J.S.; NELSON, B.L.; NICOL, D.M. Discrete-event system simulation. 3 ed. New Jersey: Prentice-Hall, 2000.

BENEDETTINI, O.; TJAHJONO, B. Towards an improved tool to facilitate simulation modelling of complex manufacturing systems. The International Journal of Advanced Manufacturing Technology, v. 43, n. 1-2, p. 191-199, 2009. DOI:

https://doi.org/10.1007/s00170-008-1686-Z

BERTRAND, J.W.M.; FRANSOO, J.C. Operations management research methodologies using quantitative modeling. International Journal of Operations \& Production 
Management, v. 22, n. 2, p. 241-264, 2002. DOI:

https://doi.org/10.1108/01443570210414338

BONAMIGO, A; PAIMELL, F. A.; ARBUGERI, L. A. Implementação do VSM por meio do Conceito Toyota Kata. In: CONGRESSO BRASILEIRO DE ENGENHARIA DE PRODUÇÃO, 6., 2017. Anais... Ponta Grossa, PR, 2017.

BONFANTE, M.C. Mapeamento de fluxo de valor como método para a manufatura sustentável: pesquisa-ação em uma empresa produtora de embalagens flexíveis. Dissertação (Mestrado) - Programa de Pós-Graduação em Engenharia Mecânica POSMEC, Universidade Federal de Santa Catarina - UFSC, Florianópolis, 2016.

BRETTEL, M.; BRETTEL, M.; FRIEDERICHSEN, N.; KELLER, M.; ROSENBERG, M. How virtualization, decentralization and network building change the manufacturing landscape: An Industry 4.0 Perspective. International Journal of Mechanical, Industrial Science and Engineering, v. 8, n. 1, p. 37-44, 2014.

BROWN, A.; AMUNDSON, J.; BADURDEEN, F. Sustainable value stream mapping (SusVSM) in different manufacturing system configurations: application case studies. Journal of Cleaner Production, v. 85, p. 164-179, 2014. DOI:

https://doi.org/10.1016/j.jclepro.2014.05.101

CHWIF, L.; MEDINA, A. C. Modelagem e simulação de eventos discretos: teoria e aplicações. 2. ed. São Paulo: Editora dos Autores, 2010.

CORREA, J.E.; MELLO, C.H.P.; PEREIRA, T.F. Uso de simulação de eventos discretos para avaliação de uma linha de montagem de uma empresa do ramo automotivo e os impactos do fator humano. Revista Científica e-Locução, v. 1, n. 6, 2015.

DAL FORNO, A.J.; PEREIRA, F.A.; FORCELLINI, F.A.; KIPPER, L.M. Value Stream Mapping: a study about the problems and challenges found in the literature from the past 15 years about application of Lean tools. The International Journal of Advanced Manufacturing Technology, v. 72, n. 5-8, p. 779-790, 2014. DOI: http://dx.doi.org/10.1007/s00170-014-5712-z

DE SOUZA, T.A.A. CARVALHO, E.R., MELO, A.C.S.; NUNES, D.R.L. Simulação e Lean Thinking como ferramentas para gestão de processos: uma avaliação de diferentes alternativas ao aumento da capacidade em uma empresa de cabos elétricos de alumínio. GEPROS: Gestão da Produção, Operações e Sistemas, v. 9, n. 4, p. 125, 2014. DOI: https://doi.org/10.15675/gepros.v9i4.1133

DONATELLI, A.; HARRIS, G. Combining value stream mapping and discrete event simulation. In: Proceedings of the Huntsville Simulation Conference. By the Society for Modeling and Simulation International, San Diego, CA., 2004.

EROL, S.; SCHUMACHER, A.; SIHN, W. Strategic guidance towards Industry 4.0-a threestage process model. In: International conference on competitive manufacturing. 2016.

FURIAN, N.; O'SULLIVAN, M.; WALKER, C.; VÖSSNER, S.; NEUBACHER, D. A conceptual modeling framework for discrete event simulation using hierarchical control structures.

Simulation modelling practice and theory, v. 56, p. 82-96, 2015. DOI:

https://doi.org/10.1016/j.simpat.2015.04.004 
GREASLEY, A. Using process mapping and business process simulation to support a process-based approach to change in a public sector organization. Technovation, n. 26, p.95-103, 2006. DOI: https://doi.org/10.1016/j.technovation.2004.07.008

GURUMURTHY, A.; KODALI, R. Design of Lean manufacturing systems using value stream mapping with simulation: a case study. Journal of Manufacturing Technology Management, v. 22, n. 4, p. 444-473, 2011. DOI: http://dx.doi.org/10.1108/17410381111126409

HARRELL, C.; GHOSH, B.K.; BOWDEN, R.O. Simulation using promodel. Boston: McGraw-Hill, 2011.

HERNANDEZ-MATIAS, J.C.; VIZAN, A.; PEREZ-GARCIA, J; RIOS, J. An integrated modelling framework to support manufacturing system diagnosis for continuous improvement. Robotics and computer-integrated manufacturing, v. 24, n. 2, p. 187-199, 2008. DOI: https://doi.org/10.1016/j.rcim.2006.10.003

JAMMES, F.; SMIT, H. Service-oriented paradigms in industrial automation. IEEE Transactions on industrial informatics, v. 1, n. 1, p. 62-70, 2005.

KAIHARA, T.; KATSUMURA, Y.; SUGINISHI, Y.; KADAR, B. Simulation model study for manufacturing effectiveness evaluation in crowdsourced manufacturing. CIRP Annals, v. 66 , n. 1, p. 445-448, 2017.

KAPPES, J.J. Aplicação dos conceitos de manufatura enxuta na produção de bens de capital sob encomenda: um estudo de caso de um produto do setor metal mecânico. Florianópolis: UFSC. Dissertação (Mestrado) - Programa de Pós-Graduação em Engenharia Mecânica - POSMEC, Universidade Federal de Santa Catarina - UFSC, Florianópolis, 2011.

KOLBERG, D.; ZÜHLKE, D. Lean automation enabled by industry 4.0 technologies. IFACPapersOnLine, v. 48, n. 3, p. 1870-1875, 2015. DOI:

https://doi.org/10.1016/j.ifacol.2015.06.359

KUMAR, S.; PHROMMATHED, P. Improving a manufacturing process by mapping and simulation of critical operations. Journal of Manufacturing Technology Management, v. 17, n. 1, p. 104-132, 2006. DOI: https://doi.org/10.1108/17410380610639533

LAW, A.M. Simulation-models level of detail determines effectiveness. Industrial engineering, v. 23, n. 10, p. 16-\&, 1991.

LAW, A.M.; MCCOMAS, MG. Simulation of manufacturing systems. In: Simulation Conference Proceedings, 1998. Winter. IEEE, 1998. p. 49-52.

LEE, E.A. Cyber physical systems: design challenges. In: Object oriented real-time distributed computing (isorc), 11th IEEE International Symposium on (pp. 363-369). IEEE, 2008.

LEE, E.A. Cyber-physical systems-are computing foundations adequate. In: Position paper for nsf workshop on cyber-physical systems: Research Motivation, Techniques and Roadmap. 2006. p. 1-9. 
LEE, J.; BAGHERI, B.; KAO, H. A cyber-physical systems architecture for industry 4.0-based manufacturing systems. Manufacturing Letters, v. 3, p. 18-23, 2015. DOI:

https://doi.org/10.1016/j.mfglet.2014.12.001

LIMA, D.F.S.; ALCANTARA, P.G.; SANTOS, L.C.; SILVA, L.M.F.; DA SILVA, R. M. Mapeamento do fluxo de valor e simulação para implementação de práticas Lean em uma empresa calçadista. Revista Produção Online, v. 16, n. 1, p. 366, 2016. DOI:

https://doi.org/10.14488/1676-1901.v16i1.2183

MASCARENHAS, S.A. Metodologia científica. Ed. Pearson. São Paulo: 2012.

MCDONALD, T.; VAN AKEN, E.M.; RENTES, A.F. Utilising simulation to enhance value stream mapping: a manufacturing case application. International Journal of Logistics, v. 5, n. 2, p. 213-232, 2002. DOI: http://dx.doi.org/10.1080/13675560210148696

MELLO, M.F.; PAVAN, L.A. Análise de tempos e métodos no setor de embalagem em uma empresa produtora de erva-mate. Engevista, v. 19, n. 5, p. 1198-1212, 2017. DOI: https://doi.org/10.22409/engevista.v19i5.956

MIHRAM, D.; MIHRAM, G.A. Human knowledge the role of models, metaphors, and analogy. International Journal of General System, v. 1, n. 1, p. 41-60, 1974. DOI: https://doi.org/10.1080/03081077408960747

MITROFF, I.I.; BETZ, F.; PONDY, L.R.; SAGASTI, F. On managing science in the system age: two schemas for the study of science as a whole system phenomenon. Interfaces, v.4, n.3, p.46-58, 1974. DOI: https://doi.org/10.1287/inte.4.3.46

MONTEVECHI, J.A.B.; LEAL, F.; PINHO, A.F.; COSTA, R.F.S.; OLIVEIRA, M.L.M.; SILVA, A.L.F. Conceptual modeling in simulation projects by mean adapted IDEF: an application in a Brazilian tech company. In: Winter Simulation Conference. Proceedings... Baltimore, MD, USA, 2010.

MOURTZIS, D.; PAPAKOSTAS, N.; MAVRIKIOS, D.; MAKRIS, S.; ALEXOPOULOS, K. The role of simulation in digital manufacturing: applications and outlook. International journal of computer integrated manufacturing, v. 28, n. 1, p. 3-24, 2015. DOI:

https://doi.org/10.1080/0951192X.2013.800234

MURAYAMA, R.C.B.; RESTON FILHO, J.C.; CARDOSO, M.A.P. Aplicação do Lean Manufacturing e estruturação da Indústria 4.0 em uma linha de baterias para smartphone. In: ENCONTRO NACIONAL DE ENGENHARIA DE PRODUÇÃO. 37., 2017. Anais.. Joinville, 2017.

OLIVEIRA, R.B.M.; CORRÊA, V.A.; NUNES, L.E.N.P. Mapeamento do fluxo de valor em um modelo de simulação computacional. Revista Produção Online, v. 14, n. 3, p. 837-861, 2014. DOI: http://dx.doi.org/10.14488/1676-1901.v14i3.1461

PEGDEN, C.D.; STURROCK, D.T. Rapid modeling solutions: introduction to simulation and Simio. Simio Forward Thinking, 2014.

RITZMAN, L.P.; KRAJEWSKI, L.J. Administração da produção. São Paulo: Prentice Hall, 2004. 
ROBINSON, S. Conceptual modelling for simulation Part I: definition and

requirements. Journal of the operational research society, v. 59, n. 3, p. 278-290, 2008.

DOI: https://doi.org/10.1057/palgrave.jors.2602368

ROTHER, M; SHOOK, J. Aprendendo a Enxegar: mapeando o fluxo de valor para agregar valor e eliminar o desperdício. São Paulo: Lean Institute Brasil, 2003.

RUIZ, N. An intelligent simulation environment for manufacturing systems. Computers \& Industrial Engineering, v. 76, n. Supplement C, p. 148-168, 2014. DOI:

https://doi.org/10.1016/j.cie.2014.06.013

SANDERS, A.; ELANGESWARAN, C.; WULFSBERG, J. Industry 4.0 implies Lean manufacturing: research activities in industry 4.0 function as enablers for Lean

manufacturing. Journal of Engineering and Management, v. 9, n. 3, p. 811-833, 2016.

DOI: http://dx.doi.org/10.3926/jiem.1940

SARGENT, R.G. Verification and validation of simulation models. Journal of simulation, v. 7, n. 1, p. 12-24, 2013. DOI: https://doi.org/10.1057/jos.2012.20

SHARARAH, M.A.; EL-KILANY, K.S.; EL-SAYED, A. E. Component bas

zed modeling and simulation of value stream mapping for lean production systems. In: FAIM Conference. 2010. p. 881-888.

SIBATROVA, S.V.; VISHNEVSKIY, K. Present and future of the production: integrating lean management into corporate foresight. 2016.

SILVA, C.E.S.; SALGADO, E.G.; MELLO, C.H.P.; SILVA OLIVEIRA, E.; LEAL, F. Integration of computer simulation in design for manufacturing and assembly. International Journal of Production Research, v. 52, n. 10, p. 2851-2866, 2014. DOI:

https://doi.org/10.1080/00207543.2013.853887

SILVA, G.F. Procedimento para análise de sistemas de produção compartilhada utilizando otimização baseada em simulação. Dissertação (Mestrado) - Universidade Federal de Santa Catarina - UFSC, Programa de Pós-graduação em Engenharia de Produção. Florianópolis, 2017.

SILVA, T.M. Mapeamento do fluxo de valor e simulação a eventos discretos para a redução de desperdícios em uma família de peças usinadas em uma empresa do setor automotivo. Dissertação (mestrado) - Universidade Federal de Santa Catarina, Centro Tecnológico, Programa de Pós-graduação em Engenharia Mecânica, Florianópolis, 2013.

SINGH, B.; GARG, S.K.; SHARMA, S.K. Value stream mapping: literature review and implications for Indian industry. The International Journal of Advanced Manufacturing Technology, v. 53, n. 5-8, p. 799-809, 2011. DOI: http://dx.doi.org/10.1007/s00170-010$\underline{2860-7}$

SLACK, N., CHAMBERS S., JOHNSTON R. Administração da produção. São Paulo: Atlas, 2009.

SMEW, W.; YOUNG, P.; GERAGHTY, J. Supply chain analysis using simulation, Gaussian process modelling and optimisation. International Journal of Simulation Modelling, v. 12, n. 3, p. 178-189, 2013. DOI: http://dx.doi.org/10.2507/IJSIMM12(3)4.239 
SOLDING, P.; GULLANDER, P. Concepts for simulation based value stream mapping. In: Simulation Conference (WSC), Proceedings of the 2009 Winter. IEEE, 2009. p. 2231-2237.

TANG, D. et al. Using autonomous intelligence to build a smart shop floor. The International Journal of Advanced Manufacturing Technology, v. 94, n. 5-8, p. 1597-1606, 2018. DOI: https://doi.org/10.1007/s00170-017-0459-y

TAYLOR, J.R. Introdução à análise de erros: o estudo de incertezas em medições físicas. Porto Alegre. Bookman, 2012.

TORTORELLA, G.L.; FETTERMAN, D.; GIGLIO, R.; BORGES, G. A. Implementação da Produção Enxuta e Indústria 4.0 em Empresas Brasileiras de Manufatura. Revista Empreender e Inovar, v. 1, n. 1, p. 1-18, 2018.

WANG, L.; TÖRNGREN, M.; ONORI, M. Current status and advancement of cyber-physical systems in manufacturing. Journal of Manufacturing Systems, v. 37, p. 517-527, 2015. DOI: https://doi.org/10.1016/j.jmsy.2015.04.008

WOLF, W. Cyber-physical systems. Computer, v. 42, n. 3, p. 88-89, 2009. DOI: https://doi.org/10.1109/MC.2009.81

WOLLMANN, D. Analytic Hierachy Process suportando a avaliação por pares. Revista Diálogo Educacional, v. 17, n. 52, p. 503-523, 2017. DOI: http://dx.doi.org/10.7213/1981416X.17.052.DS09

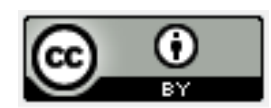

Artigo recebido em: 06/09/2018 e aceito para publicação em: 28/01/2019 DOI: http://dx.doi.org/10.14488/1676-1901.v19i1.3363 